\title{
UNIQUENESS OF AFFINE STRUCTURES ON RIEMANN SURFACES
}

\author{
JOHN T. MASTERSON
}

\begin{abstract}
Let $M$ be any compact Riemann surface of genus $g \geq 2$. It is first established that there do not exist on $M$ any generic lowdegree simple polar variations of branched affine structures having fixed nonpolar and polar branch data and fixed induced character homomorphism $\widetilde{\psi}$. Hence, these structures depend uniquely on the branch data and the homomorphism. A related result is also established concerming the nonexistence on $M$ of generic low-degree single-point variations of branched affine structures having fixed homomorphism $\widetilde{\psi}$. These results depend on the Noether and Weierstrass gaps on $M$. Corollaries are derived concerning mappings induced by sections of vector bundles of affine structures and concerning structures on an arbitrary hyperelliptic or elliptic $(g=1)$ surface $M$.
\end{abstract}

Unbranched projective structures on an arbitrary compact Riemann surface $M$ of genus $g \geq 1$ were first constructed by Gunning [2]. These structures were shown to be in one-to-one correspondence with the set of holomorphic projective connections (i.e., holomorphic quadratic differentials) $\phi$ on $M$. Analogous results for affine structures and affine connections on $M$ were also established.

A reinterpretation of a classical result of Poincaré [7] shows that these projective connections, and the corresponding projective structures, depend uniquely on the monodromy homomorphism $\psi: \pi_{1}(M) \rightarrow$ Möb associated with $\phi$. Recall that $\psi$ is the homomorphism which determines the possible global multivaluedness of some ratio $f(z)=y_{1}(z) / y_{2}(z)$ of linearly independent solutions to

Received April 20, 1991. 
the equation

$$
y^{\prime \prime}+\frac{\phi}{2} y=0
$$

on $M$. This multivaluedness is expressed by a functional equation

$$
f([\Lambda] z)=\psi([\Lambda]) \circ f(z)
$$

where $[\Lambda] \in \pi_{1}(M)$ and $f([\Lambda] z)$ represents the result of analytic continuation of $f(z)$ along the arbitrary closed loop $\Lambda$ on $M$. Note that $f$ satisfies the equation $\theta_{2} f=\phi$, where $\theta_{2}$ is the Schwarzian derivative operator. The uniqueness result just described was extended by $\mathrm{Kra}$ [3] to surfaces with a finite number of parabolic punctures.

In. view of recent results (see [4], [5], [6]) on existence of branched projective and affine structures on $M$, it would be of interest to develop an analogous uniqueness result in the branched case. This question has received little consideration to present; it is intrinsically more difficult than the corresponding unbranched question since one would expect to need some branch point and branch order data in addition to information on $\psi$ in order to prove any uniqueness conclusion.

In this paper, we restrict our attention to perhaps the most feasible uniqueness question for branched structures, namely, the problem of deriving conditions sufficient for the uniqueness of branched affine structures on an arbitrary compact Riemann surface $M$ of genus $g \geq 2$. Some of our results also extend in a natural way to surfaces of genus $g=1$.

Let $S$ be the universal covering space of $M$ with corresponding covering group $G$. Hence, $M \cong S / G$. Let $F$ be a fixed fundamental set for $G$, chosen so that $F^{0}$ is a fundamental region. The reader is referred to [4] and [5] for detailed definitions of affine and projective structures on $M$ and the associated deformations and connections for $G$. We recall that there is a canonical bijection between \{1-equivalence classes of 1-deformations $(\psi, f)$ of $G$ with affine divisor $\chi\left(=(d f)_{F}\right.$, the divisor of zeroes and poles of $d f$ in $\left.\left.F\right)\right\}$ and $\{$ branched affine structures on $M$ with affine divisor $\left.\chi^{*}=\pi^{*}(\chi)\right\}$, where $\pi^{*}$ is the map on divisors 
induced by the universal covering map $\pi: S \rightarrow M$. Note that for each affine structure on $M, \chi$ is an invariant with $\operatorname{deg}(\chi)=2 g-2$ and $\chi$ is of the form

$$
\begin{array}{ll}
\chi=\sum_{i=1}^{\ell}\left(r_{i}-l\right) p_{i}+\sum_{j=1}^{m}\left(\nu_{j}-1\right) q_{j}+\sum_{k=1}^{n}(-2) s_{k}, & p_{i}, \dot{q}_{j}, s_{k} \in F, \\
& r_{i} \in Z^{+}-\{1\}, \\
& \nu_{j} \in Z^{-}-\{-1\}, \forall i, j, k
\end{array}
$$

where $\pi\left(p_{i}\right), \pi\left(q_{j}\right)$ and $\pi\left(s_{k}\right)$ are, respectively, the nonpolar branch points of ramification orders $r_{i}$, the polar branch points of ramification orders $\left|\nu_{j}\right|$ and the simple poles of the corresponding affine structure on $M$. Each affine structure on $M$ with affine divisor divisor $\chi^{*}$ has associated branch divisor $B^{*}=\pi^{*}(B)$ on $M$, where

$$
B=\sum_{i=1}^{\ell}\left(r_{i}-1\right) p_{i}+\sum_{j=1}^{m}\left(\left|\nu_{j}\right|-1\right) q_{j}
$$

Here, $\operatorname{deg}\left(B^{*}\right)(=\operatorname{deg}(B))$ is an even integer satisfying $\operatorname{deg}\left(B^{*}\right) \geq 2 g-2$ (see [5], Lemma, 1). Additionally, each 1 -deformation $(\psi, f)$ is uniqucly determined by the locally meromorphic function $f$ and satisfies a functional equation

$$
f(A z)=\psi(A) \circ f(z), \quad \forall A \in G, \forall z \in S,
$$

with monodromy homomorphism $\psi: G \rightarrow$ Möb of the form $\psi(A)(w)=a_{A} w+$ $b_{A}(\in G A(1, C)), a_{A}, b_{A} \in C, a_{A} \neq 0, \forall A \in G . \psi$ induces an associated multiplicative character homomorphism $\tilde{\psi}: G \rightarrow C^{*}$ defined by $\widetilde{\psi}(A)=a_{A}, \forall A \in G$. Observe that the Prym differential [1] df satisfies

$$
d f(A z)=\widetilde{\psi}(A) \cdot d f(z), \quad \forall A \in G, \forall z \in S
$$

We now proceed with a detailed description of our findings.

Theorem 1. Let $M$ be any compact Riemann surface of genus $g \geq 2$. Consider any two affine structures on $M$ which both have nonpolar branch points $p_{i}^{*}, i=1, \ldots, \ell$, of ramification orders $r_{i}, r_{i} \in Z^{+}-\{1\}$, and polar branch points 
$q_{j}^{*}, j=1, \ldots, m$, of ramification orders $\left|\nu_{j}\right|, \nu_{j} \in Z^{-}-\{-1\}$, and have branch divisor $B^{*}$ satisfying

$$
\operatorname{deg}\left(B^{*}\right) \leq 3 g-2+2 \sum_{j=1}^{m}\left|\nu_{j}\right|
$$

Assume further that at least one of the simple polar divisors $D_{N}^{*}, N=1$ or 2 , of the given structures has multiple $2 D_{N}^{*}$ of minimal index of specialty $i\left(2 D_{N}^{*}\right)$ and that the two structures determine 1-deformations $\left(\psi_{N}, f_{N}\right), N=1,2$, of $G$ which induce equal character homomorphisms $\left(\widetilde{\psi}_{1}=\widetilde{\psi}_{2}\right)$. Then the two affine structures are the same.

Proof. The 1-deformations $\left(\psi_{N}, f_{N}\right), N=1,2$, have affine divisors $\chi_{N}(=$ $\left.\left(d f_{N}\right)_{F}\right), N=1,2$, given by

$$
\chi_{N}=\sum_{i=1}^{\ell}\left(r_{i}-1\right) p_{i}+\sum_{j=1}^{m}\left(\nu_{j}-1\right) q_{j}-2 D_{N}, \quad N=1,2,
$$

where $\pi\left(p_{i}\right)=p_{i}^{*}, \pi\left(q_{j}\right)=q_{j}^{*}$ and $\pi^{*}\left(D_{N}\right)=D_{N}^{*}, N=1,2$. The proof of Lemma 1 of [5] implies that

$$
\operatorname{deg}\left(B^{*}\right)=2 g-2+2 \cdot \operatorname{deg}\left(D_{N}\right)+2 \sum_{j=1}^{m}\left|\nu_{j}\right|, \quad N=1,2
$$

Equation (2) and inequality (1) together imply the inequality

$$
\operatorname{deg}\left(2 D_{N}^{*}\right) \leq g, \quad N=1,2
$$

(equivalently, $\left.\operatorname{deg}\left(D_{N}^{*}\right) \leq g / 2, N=1,2\right)$. The minimality of $i\left(2 D_{N}^{*}\right), N=1$ or 2 , and inequality (3), together with the Riemann-Roch theorem and the Noether gap theorem [1], imply that $i\left(2 D_{N}^{*}\right)=g-\operatorname{deg}\left(2 D_{N}^{*}\right), N=1$ or 2 , and that $2 D_{N}^{*}$ and all its nontrivial proper subdivisors are Noether gaps.

Define $h=d f_{2} / d f_{1}$. Then $h$ has divisor of zeroes and poles in $F$ given by $(h)_{F}=2 D_{2}-2 D_{1}$. Furthermore, $h$ is an automorphic function for $G$ (that is, $h(A z)=h(z), \forall A \in G, \forall z \in S)$, since $\widetilde{\psi}_{1}=\widetilde{\psi}_{2}$. Moreover, this observation and 
the previous conclusion concerning Noether gaps together imply that $2 D_{2}=2 D_{1}$. Hence, $h \equiv c \in C$. Consequently, $d f_{2} \equiv c d f_{1}, f_{2} \equiv c f_{1}+d$ for some constant $d$, and the deformations $\left(\psi_{N}, f_{N}\right), N=1,2$, determine the same affine structure on $M$. II

Remark 1 . In view of the inequality $\operatorname{deg}\left(D_{N}^{*}\right) \leq g / 2, N=1,2$, and the fact that minimality of the index of specialty holds for generic divisors of the form $2 D_{N}^{*}$ on $M$, Theorem 1 can be paraphrased as an assertion of the nonexistence on $M$ of generic low-degree simple polar variations of affine structures having fixed branch data and fixed character homomorphism $\tilde{\psi}$. Also, it is clear that varying the generic low-degree simple polar divisor and simultaneously fixing the branch data produces affine structures with globally varying homomorphism $\tilde{\psi}$.

Now consider affine structures on $M$ having no polar branch points and let the (fixed) branch divisor $B^{*}\left(=\Sigma_{i=1}^{\ell}\left(r_{i}-1\right) p_{i}^{*}\right)$ satisfy

$$
2 g-2 \leq \operatorname{deg}\left(B^{*}\right) \leq 4 g-2
$$

In this case, Theorem 1 of [5] asserts that for each (simple polar) divisor $D^{*}=$ $\sum_{k=1}^{n} s_{k}^{*}$ with $s_{k}^{*} \neq p_{i}^{*}, \forall i, k$, and with minimal index of specialty $i\left(D^{*}\right)$ (i.e., $i\left(D^{*}\right)=g-n$, where $\left.n=\left(\operatorname{deg}\left(B^{*}\right)-(2 g-2)\right) / 2\right)$, there exists a $g-n$ dimensional linear manifold of 1-connections (or, equivalently, of affine structures) having (fixed) affine divisor $\chi^{*}=B^{*}-2 D^{*}$. Furthermore, if $2 g-2<\operatorname{deg}\left(B^{*}\right)<$ $4 g-2$ and $X$ is the manifold of all divisors $D^{*}$ satisfying the abovementioned conditions, then these affine structures for varying $D^{*} \in X$ and fixed $B^{*}$ form a complex analytic vector bundle $E$ of rank $g-n$ over $X$ (see Corollary 1 of [5]). Consequently, Theorem 1 and Remark 1 together with Corollary 1 of [5] have the following obvious

Corollary 1. For affine structures having only nonpolar branch points, let

$$
2 g-2<\operatorname{deg}\left(B^{*}\right) \leq 3 g-2
$$

(or, equivalently, $\operatorname{deg}\left(D^{*}\right) \leq g / 2$ ) and let $X^{\prime}$ be the submanifold of $X$ for which $i\left(2 D^{*}\right)$ is minimal. Then for each section $s: X^{\prime} \rightarrow E$ of the abovementioned 
vector bundle (restricted from $X$ to $X^{\prime}$ ), the induced mapping

$$
\tilde{s}: X^{\prime} \rightarrow\left\{\begin{array}{l}
\text { character homomorphisms } \tilde{\psi} \text { for affine structures } \\
\text { associated to } e \in E \text { which project to any } \mathbb{D}^{*} \in X^{\prime}
\end{array}\right\}
$$

is one-to-one.

Theorem 2. Let $M$ be any compact Riemann surface of genus $g \geq 2$. Consider any two affine structures on $M$ having the same nonpolar and polar branch points of the same ramification orders and having the same simple poles, with one possible pair of exceptional branch points or simple poles $q_{N}^{*}, N=1,2$, corresponding to the two structures. Assume further that the two structures determine 1-deformations $\left(\psi_{N}, f_{N}\right), N=1,2$, of $G$ which induce equal character homomorphisms $\left(\widetilde{\psi}_{1}=\widetilde{\psi}_{2}\right)$. For the points $q_{N}^{*}, N=1,2$, let $\nu \in Z-\{0,1\}$ be the common ramification order (or, respectively, common negative polar order) if $q_{N}^{*}, N=1,2$, are nonpolar branch points (or, respectively, simple polar or polar branch points), Then:

(1) the conditions $|\nu-1| \leq g$ and $q_{1}^{*} \in M-\{$ Weierstrass points on $M\}$ together imply that the two affine structures are the same,

(2) the conditions $|\nu-1|<2 g, q_{1}^{*} \in\{$ Weierstrass points on $M\}$ and $|\nu-1| \in$ $\left\{\right.$ Weierstrass gaps for $\left.q_{1}^{*}\right\}$ together imply that the two affine structures are the same,

(3) the conditions $|\nu-1| \leq g, q_{1}^{*} \in\{$ Weierstrass points on $M\}$ and $|\nu-1| \in$ $\left\{\right.$ Weierstrass non-gaps for $\left.q_{1}^{*}\right\}$ together imply that $q_{2}^{*} \in V=\{$ Weierstrass points on $M$ for which $|\nu-1|$ is a non-gap\} and that an upper bound on the number of choices for the second affine structure is $\operatorname{card}(V)$.

Remark 2. In analogy with Remark 1 , we can paraphrase cases (1) and (2) of Theorem 2 as an assertion of the nonexistence on $M$ of generic low-degree single-point variations of affine structures having fixed character homomorphism $\widetilde{\psi}$. Case (3) is a discrete exception to this nonexistence.

Proof. As in the proof of Theorem 1 , the function $h\left(=d f_{2} / d f_{1}\right)$ is an automorphic function for $G$. Furthermore, $h$ has divisor of zeroes and poles in $F$ 
given by $(h)_{F}=(\nu-1) q_{2}-(\nu-1) q_{1}$, where $q_{i} \in F$ and $\pi\left(q_{i}\right)=q_{i}^{*}$ for $i=1,2$.

In cases (1) and (2), $|\nu-\hat{1}|$ is a gap for $q_{1}^{*}$. Hence, $q_{1}=q_{2}$ and $h \equiv c \in C$. Consequently, as in the proof of Theorem 1, the two affine structures are the same.

In case (3), $|\nu-1|$ is not a gap for $q_{1}^{*}$, but, by the Weierstrass gap theorem $[1],|\nu-1|$ is a gap for all $q_{2}^{*} \in\{$ non-Weierstrass points on $M\} \cup\{$ Weierstrass points not in $V\}$. Hence, $q_{2}^{*} \in V$. In fact, the function $h$ is determined up to a constant multiple by each $q_{2}^{*} \in V$. Consequently, each $q_{2}^{*} \in V$ determines at most one affine structure having affine divisor $\chi_{2}^{*}=\chi_{1}^{*}+\pi^{*}\left((h)_{F}\right)$ and character homomorphism $\widetilde{\psi}_{2}=\widetilde{\psi}_{1}$. Such a structure exists iff the function $f_{2}: S \rightarrow \hat{C}$ defined by $f_{2}=\int h \cdot d f_{1}$ is meromorphic on $S$ (or, equivalently, determines a 1-deformation of $G$ ), as opposed to being locally multivalued on $S$. Therefore, $\operatorname{card}(V)$ is an upper bound on the number of distinct affine structures in this case. II

Theorem 2 has the following

Corollary 2. Let $M$ be any compact Riemann surface of genus $g \geq 2$. Consider any two affine structures on $M$ with the same induced character homomorphism, common nonpolar and polar branch points and orders, and common simple poles with one possible pair of exceptional simple poles $s_{N}^{*}, N=1,2$ (with $|\nu-1|=2)$, corresponding to the two structures.

Then either

(1) $s_{1}^{*}$ is not a hyperelliptic Weierstrass point and the two affine structures are the same, or

(2) $s_{1}^{*}$ is a hyperelliptic Weierstrass point and there are at most $2 g+2$ choices for the second affine structure.

Proof. The conclusions follow immediately from Theroem 2. ॥

Although a surface of genus one does not possess Weierstrass points, the fact that hyperelliptic and elliptic curves have the respective algebraic representions

$$
w^{2}=\left(z-e_{1}\right)\left(z-e_{2}\right) \cdots\left(z-e_{2 g+2}\right)
$$


and.

$$
w^{2}=\left(z-e_{1}\right)\left(z-e_{2}\right)\left(z-e_{3}\right)\left(z-e_{4}\right)
$$

suggests that there is an analogue of Corollary 2 for tori. In fact, we easily obtain

Corollary 3. Let $M$ be any compact Riemann surface of genus $g=1$ and let $\mathbb{L}=<m w_{1}+n w_{2}, \forall(m, n) \in Z \times Z ; \operatorname{Im}\left(w_{2} / w_{1}\right)>0>$ be a lattice for which $M=C /<z \rightarrow z+w, \forall w \in \mathbb{L}>$. Consider any two affine structures on $M$, restricted as in the assumptions of Corollary 2. Define $s_{N}=\pi^{-1}\left(s_{N}^{*}\right), N=1,2$, and $w_{3}=w_{1}+w_{2}$.

Then there are at most four choices for the given affine structures. Furthermore, these two affine structures are the same iff $s_{1} \equiv s_{2} \bmod \mathbb{L}$ and are inequivalent iff $s_{1} \equiv\left(s_{2}+w_{i} / 2\right) \bmod \mathbb{L}$ for $i=1,2$ or 3 .

\section{References}

[1] Farkas, H. M. and I. Kra, "Riemann Surfaces", Springer-Verlag, New York, 1980.

[2] Gunning, R. C., "Special Coordinate Coverings of Riemann Surfaces", Math. Ann., 170, 67-86 (1966).

[3] Kra, 'T., "A Generalization of a Theorem of Poincaré", Proc. Am. Math. Soc., 27, 299-302 (1971).

[4] Mandelbaum, R., "Branched Structures on Riemann Surfaces", Trans. Am. Math. Soc., 163, 261-275 (1972).

[5] Masterson, J. T., "Branched Affine and Projective Structures on Compact Riemann Surfaces", Indag. Math., 91, 309-319 (1988).

[6] Masterson, J. T., "Branched Structures Associated with Lamés Equation", Arkiv för Math., 28, 131-137 (1990).

[7] Poincaré, H., "Sur les groupes des équations linéaires", Acta Math., 4, 201-312 (1884).

Dept. of Math./Comp. Sci., Seton Hall University, South Orange, New Jersey 07079, U.S.A. 